Supplementary Information for the manuscript:

\title{
Energy minimization of crystal structures containing flexible molecules
}

\section{P.G. Karamertzanis, S.L. Price}

Table S1 demonstrates the quality of reproductions of the racemic salt as well as the enantiomorphous structures for the system 1-phenylethylammonium 2-phenylpropanoate considered in the text. It also demonstrates that including the carboxylate angle $\left(\theta_{5}\right)$ in the flexible degrees of freedom leads to a small improvement in the reproduction quality. This improvement is not very significant and hence the carboxylate angle was determined by isolated-ion ab initio calculations for all refinements discussed in the text.

The changes in the cell geometry during the DMAflex refinement of the 1phenylethylammonium 2-phenylpropanoate putative rigid-body minima are summarized in Tables S2 and S3. The cell volume for both the p- and n-salt is reduced on average by only $2 \%$, whilst the changes in lattice lengths are more significant, exceeding in several cases $1 \AA$. This suggests that, although the rigid-body minima were sufficiently closed-packed structures, modelling the effect of packing forces on the ion conformations may lead to packing rearrangements in the unit cell, which concurs with the significant lattice energy stabilization observed. Given the ionic character of this system, we investigated the effect of these rerearrangements on the hydrogen bonding geometries. In the majority of the rigid-body minima the ammonium group was hydrogen bonded to three oxygen atoms belonging to different carboxylate groups, forming $R_{4}^{3}(10)$ rings similar to those found in the known structures (Bull.Chem.Soc.Jpn., 77, 79-86, 2004). The strength of these bonds can be assessed by the $(\mathrm{N}-) \mathrm{H}^{\cdots} \mathrm{O}(=\mathrm{C})$ distance and their overall geometric similarity with the ideal hydrogen bond geometry formed by a carbonyl acceptor and amine donor. The majority of $\mathrm{N}-\mathrm{H} \cdots \mathrm{O}=\mathrm{C}$ hydrogen bonds have almost linear $\mathrm{N}-\mathrm{H} \cdots \mathrm{O}$ (angle close to $180^{\circ}$ ) arrangements and an increased tendency for the carbonyl oxygen atom to form hydrogen bonds in the direction of its lone pair (angle equal to $120^{\circ}$ ). Tables S2 and S3 show that in the case of the diastereomeric salt system, the conformational changes during the DMAflex refinement improve the geometric characteristics of the hydrogen bonds for all rigid-body minima refinements.

The average all-atom RMS deformation of the anion and cation from their in vacuo geometries due to the crystalline forces are 0.214 and $0.164 \AA$ respectively for the p-salt and 0.240 and $0.168 \AA$ for the $n$-salt. In the majority of minima the ion conformations concentrate in a narrow region of conformation space (bottom-right corner of Figure 5a and bottom-left corner of Figure 5b in reference J.Phys.Chem.B., 109, 17134-17150, 2005), which also includes the majority of structures in the CSD and suggests that these ion conformations can 
systematically achieve more efficient packing arrangements, although it is also possible that some bias has been introduced by the choice of ion conformations for the rigid-body searches.

Table S1 Details for the reproduction of the known forms of 1-phenylethylammonium 2phenylpropanoate (conventional unit cells reported)

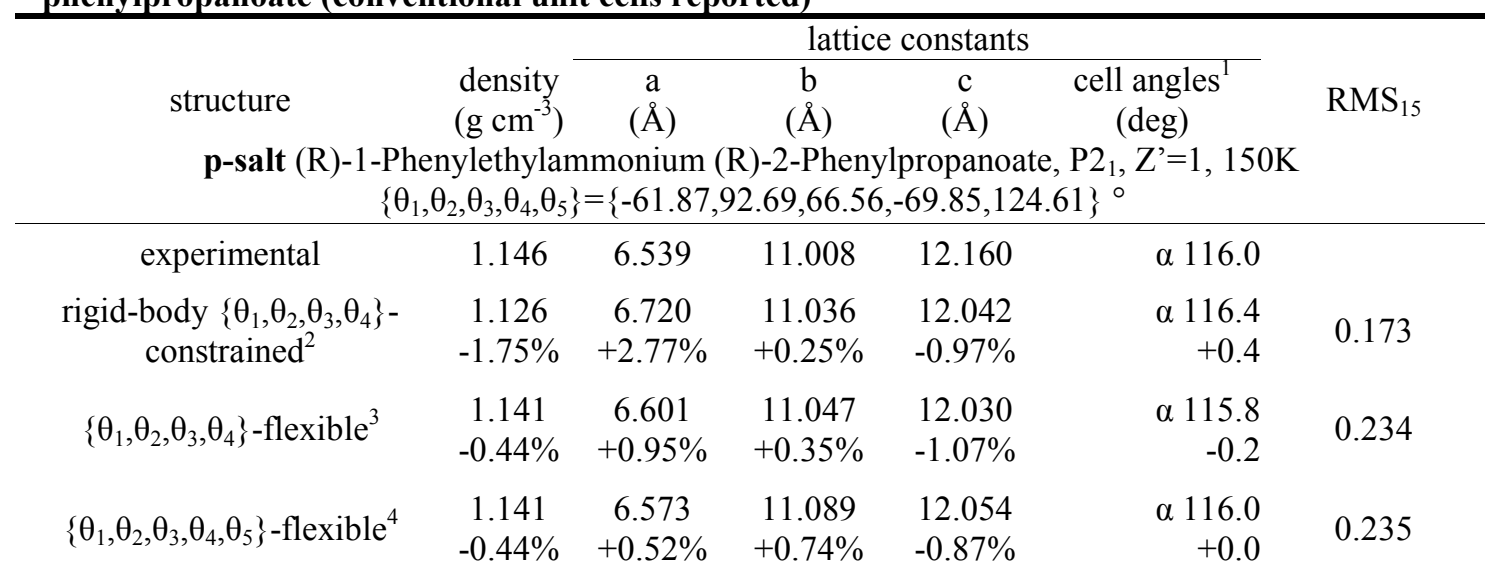

n-salt I (R)-1-Phenylethylammonium (S)-2-Phenylpropanoate, $\mathrm{P} 2{ }_{1} 2_{1} 2_{1}, \mathrm{Z}^{\prime}=1$, Room Temperature, $\left\{\theta_{1}, \theta_{2}, \theta_{3}, \theta_{4}, \theta_{5}\right\}=\{71.13,-73.28,69.28,-56.08,122.10\}^{\circ}$

\begin{tabular}{|c|c|c|c|c|c|c|}
\hline experimental & 1.179 & 5.797 & 15.444 & 17.073 & - & \\
\hline $\begin{array}{l}\text { rigid-body }\left\{\theta_{1}, \theta_{2}, \theta_{3}, \theta_{4}\right\}- \\
\text { constrained }^{2}\end{array}$ & $\begin{array}{c}1.158 \\
-1.78 \%\end{array}$ & $\begin{array}{c}6.052 \\
+4.40 \%\end{array}$ & $\begin{array}{l}15.463 \\
+0.12 \%\end{array}$ & $\begin{array}{l}16.636 \\
-2.56 \%\end{array}$ & - & 0.211 \\
\hline$\left\{\theta_{1}, \theta_{2}, \theta_{3}, \theta_{4}\right\}$-flexible ${ }^{3}$ & $\begin{array}{c}1.158 \\
-1.78 \%\end{array}$ & $\begin{array}{c}6.104 \\
+5.30 \%\end{array}$ & $\begin{array}{r}15.608 \\
+1.06 \%\end{array}$ & $\begin{array}{l}16.344 \\
-4.27 \%\end{array}$ & - & 0.382 \\
\hline$\left\{\theta_{1}, \theta_{2}, \theta_{3}, \theta_{4}, \theta_{5}\right\}$-flexible ${ }^{5}$ & $\begin{array}{c}1.164 \\
-1.27 \%\end{array}$ & $\begin{array}{r}6.039 \\
+4.17 \%\end{array}$ & $\begin{array}{c}15.597 \\
+0.99 \%\end{array}$ & $\begin{array}{l}16.441 \\
-3.70 \%\end{array}$ & - & 0.336 \\
\hline
\end{tabular}

n-salt II (R)-1-Phenylethylammonium (R)-2-Phenylpropanoate, $\mathrm{P} 22_{1} 2_{1} 2_{1}, \mathrm{Z}^{\prime}=1$, Room Temperature,

\begin{tabular}{|c|c|c|c|c|c|c|}
\hline experimental & 1.121 & 5.941 & 15.469 & 17.501 & \multicolumn{2}{|l|}{-} \\
\hline $\begin{array}{l}\text { rigid-body }\left\{\theta_{1}, \theta_{2}, \theta_{3}, \theta_{4}\right\}- \\
\text { constrained }^{2}\end{array}$ & $\begin{array}{c}1.103 \\
-1.61 \%\end{array}$ & $\begin{array}{r}6.212 \\
+4.56 \%\end{array}$ & $\begin{array}{r}15.627 \\
+1.02 \%\end{array}$ & $\begin{array}{l}16.825 \\
-3.86 \%\end{array}$ & - & 0.287 \\
\hline$\left\{\theta_{1}, \theta_{2}, \theta_{3}, \theta_{4}\right\}$-flexible ${ }^{3}$ & $\begin{array}{c}1.118 \\
-0.27 \%\end{array}$ & $\begin{array}{r}6.243 \\
+5.08 \%\end{array}$ & $\begin{array}{r}15.578 \\
+0.70 \%\end{array}$ & $\begin{array}{l}16.576 \\
-5.29 \%\end{array}$ & 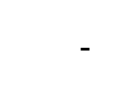 & 0.349 \\
\hline$\left\{\theta_{1}, \theta_{2}, \theta_{3}, \theta_{4}, \theta_{5}\right\}$-flexible ${ }^{6}$ & $\begin{array}{l}1.119 \\
-0.18 \%\end{array}$ & $\begin{aligned} & 6.215 \\
+ & 4.61 \%\end{aligned}$ & $\begin{array}{r}15.576 \\
+0.69 \%\end{array}$ & $\begin{array}{l}16.646 \\
-4.89 \%\end{array}$ & - & 0.332 \\
\hline \multicolumn{7}{|c|}{$\begin{array}{c}\text { racemic (R,S)-1-Phenylethylammonium }(\mathrm{R}, \mathrm{S})-2-\mathrm{Phenylpropanoate,} \mathrm{P} 2_{1} / \mathrm{c}, \mathrm{Z}^{\prime}=1,100 \mathrm{~K} \\
\left\{\theta_{1}, \theta_{2}, \theta_{3}, \theta_{4}, \theta_{5}\right\}=\{77.16,-97.42,56.05,-69.21,123.73\}^{\circ} \text { for the } \mathrm{R}-\mathrm{R} \text { pair }\end{array}$} \\
\hline experimental & 1.093 & 6.091 & 15.273 & 17.875 & $\beta 97.4$ & \multirow[b]{2}{*}{0.240} \\
\hline $\begin{array}{l}\text { rigid-body }\left\{\theta_{1}, \theta_{2}, \theta_{3}, \theta_{4}, \theta_{5}\right\}- \\
\quad \text { constrained }\end{array}$ & $\begin{array}{r}1.094 \\
+0.09 \%\end{array}$ & $\begin{array}{r}6.206 \\
+1.89 \%\end{array}$ & $\begin{array}{l}15.198 \\
-0.49 \%\end{array}$ & $\begin{array}{l}17.641 \\
-1.31 \%\end{array}$ & $\begin{array}{r}\beta \\
\quad 98.1 \\
+0.7\end{array}$ & \\
\hline$\left\{\theta_{1}, \theta_{2}, \theta_{3}, \theta_{4}, \theta_{5}\right\}$-flexible ${ }^{7}$ & $\begin{aligned} & 1.125 \\
+ & 2.93 \%\end{aligned}$ & $\begin{aligned} & 6.282 \\
+ & 3.14 \%\end{aligned}$ & $\begin{array}{l}15.092 \\
-1.19 \%\end{array}$ & $\begin{array}{c}17.088 \\
-4.40 \%\end{array}$ & $\begin{array}{r}\beta 98.6 \\
+1.2\end{array}$ & 0.381 \\
\hline \multicolumn{7}{|c|}{$\begin{array}{l}{ }^{1} \text { lattice angles constrained by space group symmetry are omitted. } \\
2 \text { rigid body minimization with the ion conformations derived by an MP2/6-31G(d,p) optimization with } \\
\text { the indicated torsion angles constrained to their experimental values (J.Phys.Chem.B., 109, 17134-17150, } \\
2005 \text { ) } \\
3 \text { the values of }\left\{\theta_{1}, \theta_{2}, \theta_{3}, \theta_{4}\right\} \text { at the minimum are shown in Tables } 3 \& 4 \text { of the manuscript. } \\
4,5,6,7 \text { the values of }\left\{\theta_{1}, \theta_{2}, \theta_{3}, \theta_{4}, \theta_{5}\right\} \text { at the minimum are }\left\{-52.37^{\circ}, 87.53^{\circ}, 74.68^{\circ},-64.78^{\circ}, 126.42^{\circ}\right\}, \\
\left\{62.05^{\circ},-59.51^{\circ}, 74.92^{\circ},-62.13^{\circ}, 126.20^{\circ}\right\},\left\{73.88^{\circ},-95.77^{\circ}, 66.39^{\circ},-74.86^{\circ}, 126.97^{\circ}\right\} \text { and }\left\{70.16^{\circ},-\right. \\
\left.94.65^{\circ},-69.59^{\circ},-66.16^{\circ}, 126.99^{\circ}\right\} \text { (R-ions) respectively. We note that the HF/6-31G(d,p) minimum } \\
\text { value for } \theta_{5} \text { is } 129.56^{\circ} \text {. }\end{array}$} \\
\hline
\end{tabular}


Table S3 Changes in cell geometry and average hydrogen bond geometries of rigid-body n-salt 1phenylethylammonium 2-phenylpropanoate minima after the DMAflex refinement. Rows in bold correspond to known forms.

\begin{tabular}{|c|c|c|c|c|c|c|c|c|c|c|c|c|c|}
\hline \multirow{3}{*}{$\begin{array}{l}\text { flexible ion } \\
\text { search } \\
\text { rank, } \\
\text { hydrogen } \\
\text { bond motif }\end{array}$} & \multirow{3}{*}{$\Delta \hat{V}^{3}$} & \multicolumn{4}{|c|}{ changes in cell geometry ${ }^{1}$} & \multicolumn{8}{|c|}{$\mathrm{NH}^{\cdots} \mathrm{O}=\mathrm{C}$ hydrogen bond geometry ${ }^{2}$} \\
\hline & & \multirow{2}{*}{$\begin{array}{c}\Delta \mathrm{a} \\
(\AA)\end{array}$} & \multirow{2}{*}{$\begin{array}{l}\Delta \mathrm{b} \\
(\AA)\end{array}$} & \multirow{2}{*}{$\begin{array}{l}\Delta \mathrm{c} \\
(\AA)\end{array}$} & \multirow{2}{*}{$\begin{array}{l}\Delta \alpha, \Delta \beta, \Delta \gamma^{4} \\
\quad(\operatorname{deg})\end{array}$} & \multirow{2}{*}{$\begin{array}{c}\mathrm{NH} \cdots \mathrm{O} \\
(\AA) \\
\text { mean }\end{array}$} & \multicolumn{3}{|c|}{$\begin{array}{lc}\mathrm{NH}^{\cdots} \mathrm{O} & \mathrm{C}=\mathrm{O} \cdots \mathrm{H} \\
(\mathrm{deg}) & (\mathrm{deg})\end{array}$} & \multirow{2}{*}{$\begin{array}{c}\mathrm{NH} \cdots \mathrm{O} \\
(\AA) \\
\text { mean }\end{array}$} & \multirow{2}{*}{$\begin{array}{c}\mathrm{NH}^{\cdots} \mathrm{O} \\
(\mathrm{deg}) \\
\text { mean }\end{array}$} & \multicolumn{2}{|c|}{$\begin{array}{c}\mathrm{C}=\mathrm{O} \cdots \mathrm{H} \\
\quad(\mathrm{deg})\end{array}$} \\
\hline & & & & & & & & mean & st.dev. & & & mean & st.dev. \\
\hline $3, R_{4}^{3}(10)$ & -0.69 & $-\mathbf{0 . 0 3 7}$ & $7-\mathbf{0 . 0 7 9}$ & 0.002 & - & 1.800 & 154.47 & 116.83 & 6.16 & 1.741 & 161.69 & 122.67 & 1.10 \\
\hline $7, R_{4}^{3}(10)$ & -1.80 & 0.837 & -0.354 & -0.008 & - & 1.820 & 154.68 & 130.21 & 31.32 & 1.808 & 155.63 & 123.19 & 27.93 \\
\hline $4, R_{4}^{3}(10)$ & -7.34 & 1.927 & -0.161 & -2.968 & - & 1.765 & 154.09 & 118.33 & 3.70 & 1.740 & 161.90 & 122.48 & 0.05 \\
\hline $10, R_{4}^{3}(10)$ & -0.44 & 0.029 & 0.234 & -0.164 & - & 1.746 & 152.92 & 123.24 & 13.74 & 1.721 & 162.31 & 123.08 & 7.78 \\
\hline $12, R_{4}^{3}(10)$ & -1.50 & -0.058 & -0.036 & -0.031 & $\Delta \beta 0.814$ & 1.756 & 152.77 & 120.90 & 10.64 & 1.730 & 159.48 & 123.82 & 9.02 \\
\hline 15 & -0.50 & 0.799 & -0.156 & -0.266 & - & & & & & & & & \\
\hline $19, R_{4}^{3}(10)$ & -1.80 & -0.374 & +-0.060 & 0.066 & - & 1.827 & 154.60 & 124.26 & 23.49 & 1.828 & 159.02 & 126.59 & 27.64 \\
\hline $6, R_{4}^{3}(10)$ & -0.20 & 0.575 & 0.499 & -0.487 & - & 1.804 & 154.07 & 132.27 & 26.52 & 1.741 & 161.52 & 129.20 & 12.94 \\
\hline $\begin{array}{c}8 \\
13\end{array}$ & $\begin{array}{l}-0.29 \\
-1.50\end{array}$ & $\begin{array}{l}-0.603 \\
-0.140\end{array}$ & & & - & & & & & & & & \\
\hline $14, R_{4}^{3}(10)$ & -1.73 & 0.076 & -0.105 & 0.146 & $\Delta \beta-0.333$ & 1.751 & 153.50 & 120.03 & 6.40 & 1.720 & 163.63 & 120.82 & 7.74 \\
\hline $1, R_{4}^{3}(10)$ & -2.84 & 0.309 & $\mathbf{- 0 . 7 9 5}$ & -0.008 & - & 1.876 & 155.90 & 106.31 & 13.41 & 1.725 & 162.88 & 120.66 & 6.14 \\
\hline $11, R_{6}^{5}(16)$ & -1.21 & -0.448 & -0.259 & 0.165 & - & 1.942 & 151.03 & 124.12 & 25.13 & 1.963 & 152.90 & 116.55 & 22.05 \\
\hline $2, R_{4}^{3}(10)$ & -5.20 & 0.485 & -0.142 & -0.073 & $\Delta \beta 2.126$ & 1.790 & 155.26 & 123.31 & 15.94 & 1.745 & 162.45 & 125.78 & 8.29 \\
\hline $9, R_{4}^{3}(10)$ & -4.52 & 0.276 & 0.396 & -0.575 & - & 1.806 & 150.57 & 126.76 & 34.14 & 1.756 & 158.37 & 123.93 & 23.12 \\
\hline $16, R_{4}^{3}(10)$ & -2.01 & -0.388 & -0.157 & 0.317 & - & 1.797 & 160.52 & 131.13 & 11.85 & 1.771 & 165.49 & 127.19 & 9.81 \\
\hline $17, R_{6}^{5}(16)$ & -1.58 & 0.135 & -0.390 & -0.009 & $\Delta \beta-0.533$ & 1.931 & 151.66 & 122.40 & 23.81 & 1.953 & 152.04 & 118.11 & 22.45 \\
\hline $18, R_{6}^{5}(16)$ & -0.58 & 0.114 & -0.198 & -0.074 & $\Delta \beta-0.516$ & 1.941 & 150.45 & 124.52 & 25.57 & 1.953 & 151.81 & 118.14 & 22.51 \\
\hline $5, R_{4}^{3}(10)$ & -4.28 & -0.322 & 0.179 & -0.227 & - & 1.752 & 160.61 & 126.30 & 20.53 & 1.720 & 166.45 & 121.74 & 3.82 \\
\hline \multirow[t]{2}{*}{$20, R_{4}^{3}(10)$} & -1.02 & 0.215 & -0.270 & 0.306 & $\Delta \beta-0.553$ & 1.764 & 150.16 & 118.96 & 10.35 & 1.719 & 165.52 & 117.86 & 8.66 \\
\hline & & & & & average & 1.816 & 153.96 & 122.93 & 17.806 & 1.784 & 160.18 & 122.46 & 13.00 \\
\hline \multicolumn{14}{|c|}{${ }^{1}$ flexible ion minimum - rigid-body lattice energy minimum } \\
\hline \multicolumn{14}{|c|}{$\begin{array}{l}{ }^{5} \text { lattice angles constrained by space group symmetry are omitted. } \\
\text { in the "ideal" hydrogen bond angle geometry the amine hydrogen is in the direction of the oxygen lone pair, i.e. } \mathrm{C}=\mathrm{O} \cdots \mathrm{H} \\
\text { angle } 120^{\circ} \text {. Because ideal mean } \mathrm{C}=\mathrm{O} \cdots \mathrm{H} \text { values may be obtained when the three } \mathrm{C}=\mathrm{O} \cdots \mathrm{H} \text { angles are widely different than } \\
120^{\circ} \text {, the standard deviation for the three bonds is also reported. }\end{array}$} \\
\hline
\end{tabular}


Table S4 Changes in cell geometry and hydrogen bond geometries of rigid-body carbamazepine minima after the DMAflex refinement. Rows in bold correspond to known forms. The hydrogen bond geometry is reported only for minima exhibiting $\mathrm{N}-\mathrm{H} \cdots \mathrm{O}=\mathrm{C}$ bonds.

\begin{tabular}{|c|c|c|c|c|c|c|c|c|c|c|c|}
\hline \multirow{3}{*}{$\begin{array}{c}\text { flexible } \\
\text { molecule } \\
\text { search rank, } \\
\text { hydrogen } \\
\text { bond motif }\end{array}$} & \multirow{2}{*}{\multicolumn{5}{|c|}{ changes in cell geometry ${ }^{1}$}} & \multicolumn{6}{|c|}{$\mathrm{NH}^{\cdots} \mathrm{O}=\mathrm{C}$ hydrogen bond geometry } \\
\hline & & & & & & & rigid-bc & & & flexible-i & \\
\hline & $\begin{array}{l}\Delta \hat{V}^{2} \\
(\%)\end{array}$ & $\begin{array}{l}\Delta \mathrm{a} \\
(\AA)\end{array}$ & $\begin{array}{l}\Delta \mathrm{b} \\
(\AA)\end{array}$ & $\begin{array}{l}\Delta \mathrm{c} \\
(\AA)\end{array}$ & $\begin{array}{l}\Delta \alpha, \Delta \beta, \Delta \gamma^{3} \\
\quad(\mathrm{deg})\end{array}$ & $\begin{array}{l}\mathrm{NH}^{\cdots} \mathrm{O} \\
(\AA)\end{array}$ & $\begin{array}{c}\mathrm{NH} \cdots \mathrm{O} \\
(\mathrm{deg})\end{array}$ & $\begin{array}{c}\mathrm{C}=\mathrm{O} \cdots \mathrm{H}^{4} \\
(\mathrm{deg})\end{array}$ & $\begin{array}{l}\mathrm{NH}^{\cdots} \mathrm{O} \\
(\AA)\end{array}$ & $\begin{array}{c}\mathrm{NH} \cdots \mathrm{O} \\
\text { (deg) }\end{array}$ & $\begin{array}{c}\mathrm{C}=\mathrm{O} \cdots \mathrm{H}^{4} \\
(\mathrm{deg})\end{array}$ \\
\hline $1, \mathrm{C} 4$ & -0.62 & 0.143 & 0.003 & -0.269 & $\Delta \beta 0.30$ & 2.243 & 131.70 & 126.18 & 2.191 & 132.45 & 128.19 \\
\hline $2, \mathrm{R}_{2}^{2}(8)$ & -1.75 & -0.145 & 0.059 & -0.055 & $\Delta \beta 0.11$ & 1.958 & 166.33 & 114.64 & 1.942 & 173.10 & 115.02 \\
\hline $8, \mathrm{C} 4$ & -0.79 & 0.117 & -0.033 & -0.230 & $\Delta \beta-0.32$ & 2.225 & 134.95 & 130.22 & 2.182 & 134.49 & 131.55 \\
\hline $10, \mathrm{C} 4$ & -1.11 & -0.139 & -0.080 & 0.083 & - & 2.006 & 154.59 & 149.15 & 2.010 & 153.72 & 146.05 \\
\hline $3, \mathrm{R}_{2}^{2}(8)$ & -0.94 & -0.042 & -0.003 & -0.171 & $\begin{array}{c}\Delta \alpha-0.82, \Delta \beta- \\
1.73, \Delta \gamma 2.18\end{array}$ & 1.899 & 175.66 & 122.96 & 1.892 & 169.38 & 125.35 \\
\hline $6, \mathrm{C} 4$ & -1.46 & 0.030 & -0.192 & 0.014 & & 2.180 & 136.57 & 144.50 & 2.115 & 136.66 & 148.70 \\
\hline $5, \mathrm{R}_{2}^{2}(8)$ & -2.25 & -0.179 & 0.083 & -0.112 & $\begin{array}{c}\Delta \alpha-1.40, \Delta \beta- \\
0.39, \Delta \gamma 0.93\end{array}$ & 1.880 & 171.62 & 114.71 & 1.914 & 166.78 & 114.54 \\
\hline $14, \mathrm{R}_{2}^{2}(8)$ & -0.94 & -0.159 & 0.027 & 0.013 & $\begin{array}{c}\Delta \alpha-1.03, \Delta \beta \\
0.23, \Delta \gamma-0.06\end{array}$ & 1.924 & 164.73 & 124.36 & 1.926 & 164.60 & 127.86 \\
\hline $7, \mathrm{R}_{2}^{2}(8)$ & -1.15 & 0.051 & -0.067 & -0.371 & $\Delta \beta 0.91$ & 1.900 & 174.75 & 121.23 & 1.897 & 168.24 & 121.45 \\
\hline $9, \mathrm{C} 4$ & -1.73 & -0.108 & 0.007 & -0.099 & $\Delta \beta 0.23$ & 2.158 & 134.29 & 149.37 & 2.099 & 138.22 & 152.48 \\
\hline $4, \mathrm{C} 2$ & -3.08 & -0.151 & -0.143 & -0.039 & - & & & & & & \\
\hline $15, \mathrm{C} 4$ & -1.23 & -0.111 & 0.057 & -0.127 & $\Delta \beta-0.19$ & 1.988 & 155.06 & 145.76 & 1.992 & 153.91 & 144.19 \\
\hline $13, \mathrm{R}_{2}^{2}(8)$ & -1.20 & -0.116 & 0.119 & -0.082 & $\begin{array}{l}\Delta \alpha-1.37, \Delta \beta- \\
0.16, \Delta \gamma-0.60\end{array}$ & 1.955 & 160.20 & 126.56 & 1.929 & 164.88 & 129.83 \\
\hline 24 , none & -1.03 & -0.095 & -0.164 & 0.084 & $\begin{array}{l}\Delta \alpha-0.28, \Delta \beta- \\
1.21, \Delta \gamma-1.26\end{array}$ & & & & & & \\
\hline $12, \mathrm{R}_{2}^{2}(8)$ & -1.82 & 0.058 & -0.149 & -0.351 & $\Delta \beta 0.77$ & 1.879 & 171.56 & 114.58 & 1.907 & 167.60 & 115.35 \\
\hline $18, \mathrm{R}_{2}^{2}(8)$ & -0.67 & 0.143 & -0.163 & -0.133 & $\Delta \beta-0.47$ & 1.953 & 170.85 & 123.63 & 1.924 & 174.40 & 124.39 \\
\hline $16, \mathrm{R}_{2}^{2}(8)$ & -1.63 & 0.018 & -0.036 & 0.544 & $\Delta \beta-1.35$ & 1.994 & 147.82 & 132.54 & 1.953 & 157.15 & 131.95 \\
\hline $21, \mathrm{R}_{2}^{2}(8)$ & -1.43 & -0.004 & -0.044 & -0.064 & $\begin{array}{l}\Delta \alpha-0.27, \Delta \beta \\
0.48, \Delta \gamma 0.47\end{array}$ & 1.908 & 167.86 & 129.45 & 1.899 & 171.18 & 129.06 \\
\hline $19, \mathrm{C} 4$ & -1.66 & -0.234 & 0.441 & -0.037 & $\Delta \beta-0.53$ & 2.168 & 124.52 & 146.70 & 2.144 & 125.60 & 143.79 \\
\hline $25, \mathrm{C} 4$ & -1.25 & -0.153 & 0.119 & -0.109 & $\Delta \beta-0.21$ & 2.002 & 160.18 & 149.70 & 2.007 & 159.33 & 148.17 \\
\hline $26, \mathrm{C} 4$ & -1.22 & -0.042 & 0.003 & -0.206 & $\Delta \beta 0.89$ & 2.073 & 155.70 & 115.58 & 2.060 & 155.13 & 113.59 \\
\hline $17, \mathrm{C} 4$ & -1.47 & -0.125 & -0.051 & -0.012 & $\Delta \beta 0.90$ & & & & & & \\
\hline $27, \mathrm{R}_{2}^{2}(8)$ & -1.09 & -0.192 & 0.146 & -0.094 & $\Delta \beta-1.25$ & 1.942 & 168.52 & 128.92 & 1.932 & 170.67 & 129.68 \\
\hline 20, $\mathrm{R}_{2}^{2}(8)$ & -1.10 & -0.048 & 0.023 & -0.268 & $\Delta \beta 0.42$ & 1.889 & 173.77 & 119.60 & 1.888 & 167.88 & 119.55 \\
\hline 11, $\mathrm{R}_{2}^{2}(8)$ & -3.22 & -0.018 & -0.121 & 0.146 & $\Delta \beta-0.70$ & 1.883 & 175.68 & 126.72 & 1.912 & 170.67 & 121.28 \\
\hline $22, \mathrm{R}_{2}^{2}(8)$ & -1.66 & -0.221 & -0.001 & 0.171 & $\Delta \beta 1.34$ & 1.871 & 173.70 & 117.34 & 1.898 & 168.14 & 116.49 \\
\hline $23, \mathrm{C} 4$ & -1.31 & -0.056 & -0.010 & -0.154 & - & 2.463 & 106.07 & 127.22 & 2.431 & 105.72 & 128.58 \\
\hline 29, C4 & -1.43 & -0.074 & -0.346 & 0.376 & - & 2.203 & 123.83 & 139.48 & 2.196 & 123.80 & 138.64 \\
\hline $28, \mathrm{C} 4$ & -1.27 & -0.068 & -0.336 & 0.039 & - & 2.083 & 155.78 & 114.52 & 2.061 & 155.06 & 113.17 \\
\hline $\begin{array}{l}3 \text { change } \\
\text { lattice ang } \\
\text { in the "id } \\
\mathrm{C}=\mathrm{O} \cdots \mathrm{H}\end{array}$ & cell & av & erage & t & . & 2.024 & 155.24 & 129.06 & 2.012 & 154.95 & 129.19 \\
\hline
\end{tabular}




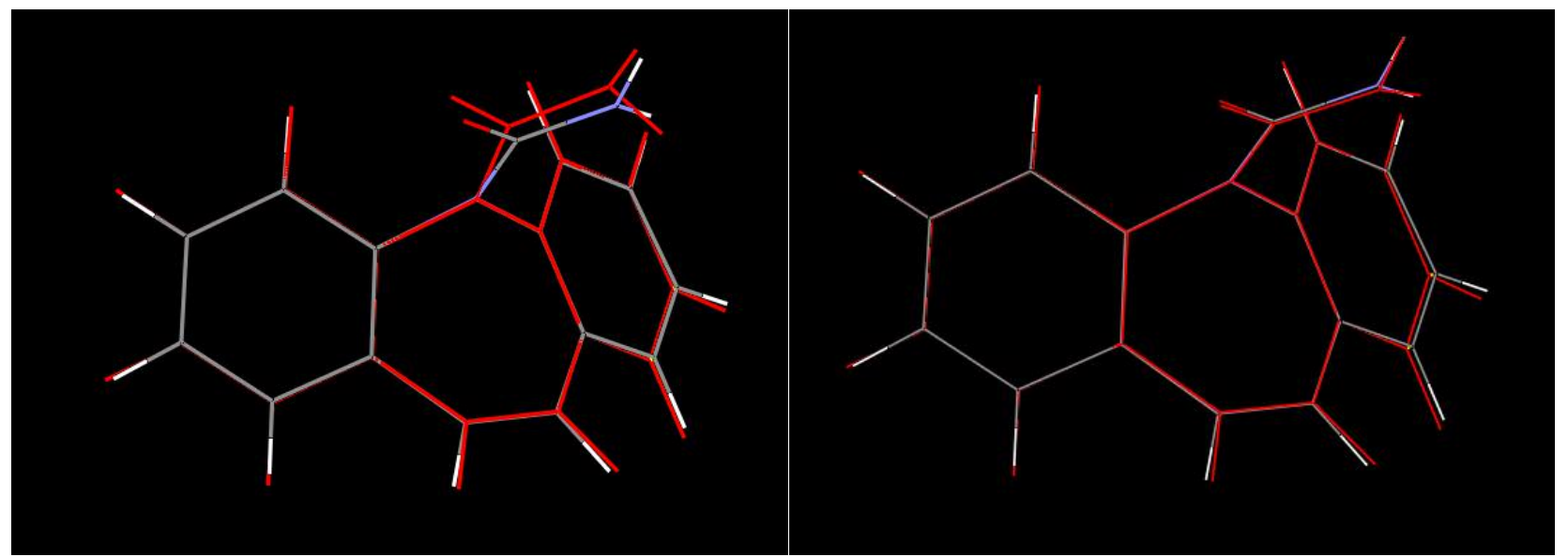

Figure S1 Conformation overlay of the experimentally determined form IV of carbamazepine (coloured by element) with the rigid-body search molecular conformation (left) and the molecular conformation after flexible-molecule refinement (right) shown in red. 